\title{
Author Correction: Fluctuations in Jupiter's equatorial stratospheric oscillation
}

Arrate Antuñano (D), Richard G. Cosentino (D), Leigh N. Fletcher, Amy A. Simon, Thomas K. Greathouse and Glenn S. Orton

Correction to: Nature Astronomy https://doi.org/10.1038/s41550-020-1165-5, published online 24 August 2020.

In the version of this Article originally published, in affiliation 5, "California Institute of Technology" was missing; this has now been corrected in all versions of the Article.

Published online: 7 April 2021

https://doi.org/10.1038/s41550-021-01362-8

() Springer Nature Limited 2021 